\title{
Nicotine enhances hepatocyte growth factor-mediated lung cancer cell migration by activating the $\alpha 7$ nicotine acetylcholine receptor and phosphoinositide kinase-3-dependent pathway
}

\author{
REMI YONEYAMA ${ }^{1,2}$, KAZUTETSU AOSHIBA ${ }^{3}$, KINYA FURUKAWA ${ }^{2}$, MAKOTO SAITO $^{2}$, \\ HIROAKI KATABA $^{2}$, HIROYUKI NAKAMURA ${ }^{3}$ and NORIHIKO IKEDA ${ }^{1}$ \\ ${ }^{1}$ Department of Thoracic Surgery, Tokyo Medical University Hospital, Shinjuku-ku, Tokyo 160-0023; \\ Departments of ${ }^{2}$ Chest Surgery and ${ }^{3}$ Respiratory Medicine, Tokyo Medical University Ibaraki Medical Center, \\ Inashiki-gun, Ibaraki 300-0395, Japan
}

Received February 17, 2015; Accepted October 30, 2015

DOI: $10.3892 / \mathrm{ol} .2015 .3930$

\begin{abstract}
Cigarette smoking not only promotes lung carcinogenesis, but it has also been demonstrated to promote the progression of lung cancer. Despite nicotine being a major component of cigarette smoke, it is not carcinogenic when acting alone. Instead, it is believed to function as a tumor promoter. Due to the fatal consequences of lung cancer being primarily associated with the processes of invasion and metastasis, the present study aimed to determine the effect of nicotine on the migratory activity of lung cancer cells. The effect of nicotine on the migration of lung cancer A549 cells was evaluated by a wound healing assay. Hepatocyte growth factor (HGF) was used as a pro-migratory stimulus. During several of the experiments, specific inhibitors of $\alpha 7$-nicotine acetylcholine receptor ( $\alpha 7$-nAchR), phosphoinositide kinase-3 (PI3K) and extracellular signal-related kinase (ERK)1/2 were included. The phosphorylation levels of Akt and ERK1/2 were examined using a cell-based protein phosphorylation assay. It was observed that nicotine did not induce cell migration by itself, but that it instead promoted HGF-induced cell migration. The effects of nicotine were inhibited by the pretreatment of the cells with the $\alpha 7$-nAchR inhibitor, methyllycaconitine, and the PI3K inhibitor, LY294002. The mitogen-activated protein kinase/ERK kinase kinase inhibitor exerted modest, but non-significant inhibitory activity on the effect of nicotine. Nicotine did not induce Akt phosphorylation by itself, but instead promoted the HGF-induced phosphorylation of Akt. It was also observed that nicotine had no effect on ERK1/2 phosphorylation. The results from the present study indicate
\end{abstract}

Correspondence to: Professor Kazutetsu Aoshiba, Department of Respiratory Medicine, Tokyo Medical University Ibaraki Medical Center, 3-20-1 Chuo, Ami-machi, Inashiki-gun, Ibaraki 300-0395, Japan

E-mail: kaoshiba@tokyo-med.ac.jp

Key words: nicotine, lung cancer, migration, hepatocyte growth factor, $\alpha 7$ nicotine acetylcholine receptor, phosphoinositide kinase-3 that nicotine, when alone, does not have a pro-migratory function, but instead enhances responsiveness to the pro-migratory stimulus emitted by HGF. The current study provides an insight into the mechanism of tumor promotion by demonstrating that nicotine and $\alpha 7$-nAchRs act in synergy with the HGF-induced PI3K/Akt signaling pathway, increasing the sensitivity of lung cancer cells to HGF, and thereby promoting cell migration, a vital step in invasion and metastasis.

\section{Introduction}

Lung cancer has one of the lowest survival rates of all types of cancer, accounting for 1.59 million mortalities in the world in 2012 (1). Smoking, particularly of cigarettes, is estimated to cause $87 \%$ of the lung cancer mortalities in men, and $70 \%$ of the lung cancer mortalities in women (2). Cigarette smoke contains numerous carcinogens, of which, polycyclic aromatic hydrocarbons and the tobacco-specific nitrosamine, 4-(methylnitrosamino)-1-(3-pyridyl)-1-butanol, are likely to serve major roles (3). However, recent evidence suggests that cigarette smoking not only promotes lung carcinogenesis, but that it also promotes the progression of lung cancer (4). For example, continued smoking during lung cancer treatment has been associated with decreased survival (5), while smoking cessation during the treatment improves the therapeutic outcome (6). Furthermore, cigarette smoking increases the risk of recurrence and the cancer-specific mortality in non-cigarette smoke-associated cancers, including prostate cancer $(7,8)$. Nicotine is one of the components of cigarette smoke that may promote cancer progression.

Whilst it does not exert direct carcinogenic activity, nicotine is commonly accepted instead as a tumor promoter (4). Recent evidence suggests that the tumor-promoting effects of nicotine result in increased proliferation, invasion, mesenchymal transition (EMT) and angiogenesis, and decreased cell death and apoptosis (4,9-17). However, the molecular mechanisms by which nicotine promotes tumor progression are not yet fully understood.

Cell motility is a critical event during the invasion and metastasis of cancer cells. During the present study, the effect 
of nicotine on the motility of lung cancer A549 cells was investigated. It was demonstrated that nicotine, by itself, does not induce A549 cell migration, but instead promotes hepatocyte growth factor (HGF)-induced migration. The tumor-promoting effect of nicotine was mediated by its binding to $\alpha 7$-nicotine acetylcholine receptors $(\alpha 7-n A c h R)$ and the subsequent upregulation of the HGF-stimulated phosphoinositide kinase-3 (PI3K)/Akt signaling pathway. The results of the present study indicate that nicotine enhances the pro-migratory effect of HGF on lung cancer cells, thereby potentially contributing to lung cancer progression.

\section{Materials and methods}

Cell culture. Lung cancer A549 cells were purchased from the American Type Culture Collection (Manassas, VA, USA). The cells were grown and maintained on bovine type I collagen-coated (Nippi, Inc., Tokyo, Japan) plates in Dulbecco's modified Eagle's medium (DMEM; Gibco ${ }^{\circledR}$; Thermo Fisher Scientific, Inc., Waltham, MA, USA), containing 10\% fetal bovine serum (FBS) at $37^{\circ} \mathrm{C}$ in a humidified incubator saturated with a gas mixture containing $5 \% \mathrm{CO}_{2}$.

In vitro wound healing assay. The migratory activity of the cells was determined by the in vitro wound healing assay (18). A549 cells were grown to confluence in 24 -well plates. The cells were serum-starved in $0.5 \%$ FBS for $48 \mathrm{~h}$ and were subsequently washed with phosphate-buffered saline (PBS). The cells were then scratched in a straight line with a sterile $200-\mathrm{ml}$ tip and washed twice with PBS. Following this, the cells were incubated in serum-free DMEM containing or lacking HGF (20 ng/ml; PromoCell GmbH, Heidelberg, Germany) and in the presence or absence of nicotine $(0.1,1$ and $10 \mu \mathrm{M}$; Wako Pure Chemical Industries, Ltd., Osaka, Japan). In several experiments, medium was added with specific inhibitors $1 \mathrm{~h}$ prior to the treatment with HGF and nicotine. The specific inhibitors included: Methyllycaconitine $(10 \mu \mathrm{M}$; Sigma-Aldrich Japan, Tokyo, Japan), an inhibitor of $\alpha 7-n$ AchR, LY294002 (5 $\mu \mathrm{M}$; Cayman Chemical Company, Ann Arbor, MI, USA), an inhibitor of PI3K, and PD98059 (5 $\mu \mathrm{M}$; Cayman Chemical Company), an inhibitor of mitogen-activated protein kinase (MAPK)/extracellular signal related kinase (ERK) kinase (MEK). Following 12 or $18 \mathrm{~h}$, the cells were fixed with $10 \%$ formalin and washed twice with PBS. Wound healing was observed with the Olympus Model IX71 Fluorescence Microscope with phase contrast (Olympus Corporation, Tokyo, Japan) equipped with a digital camera. The area of the wound was measured using image analysis software (Win Roof Version 3.5; Mitani Corporation, Fukui, Japan) on a Microsoft XP computer. A total of 5 measurements were taken from 5 fields of each well, obtained from 6 wells in each experiment.

Cell-based protein phosphorylation assay. The A549 cells were grown to $90 \%$ confluence in 96-well plates and serum-starved in DMEM with $0.5 \% \mathrm{FBS}$. The cells were subsequently treated with $\mathrm{HGF}(20 \mathrm{ng} / \mathrm{ml})$ and/or nicotine $(10 \mu \mathrm{M})$ for $2 \mathrm{~h}$, and fixed with $10 \%$ formalin. Phosphorylation of ERK1/2 and Akt protein was assayed using the phospho-ERK1/2 antibody or the phospho-Akt antibody from Fast Activated Cell-based ELISA kits (Active Motif, Carlsbad, CA, USA), according to the manufacturer's protocols. Absorbance was measured using a Benchmark Plus microplate spectrophotometer (Bio-Rad Laboratories, Inc., Hercules, CA, USA) at $450 \mathrm{~nm}$, with a reference wavelength of $655 \mathrm{~nm}$. Following this, the wells were stained with a crystal violet solution and the absorbance at $595 \mathrm{~nm}$ was measured. The protein phosphorylation levels were corrected for the cell number by dividing the optical density $(\mathrm{OD})_{450}$ reading for a given well by the $\mathrm{OD}_{595}$ reading for that well.

Statistical analysis. The statistical analyses were performed using Excel X software with the add-in software Statcel 3 (OMS Inc., Tokyo, Japan). Data are expressed as the mean \pm standard error of the mean. Statistical differences were analyzed by analysis of variance, and if the results were significant, the Tukey-Kramer test was employed as a multiple comparison post hoc test. $\mathrm{P}<0.05$ was considered to indicate a statistically significant difference.

\section{Results}

Nicotine promotes the migration of HGF-treated A549 cells. The migration of cancer cells is a vital step in cancer invasion and metastasis. To determine the effect of nicotine on A549 cell migration, a wound healing assay was conducted, which is typically considered to be a simple and reliable test for the evaluation of cell motility. During the assay, the cells were maintained in serum-free medium in order to avoid any potential modulatory effect that the serum may have on cell motility. As presented in Fig. 1, it was observed that nicotine had no effect on cell migration towards the scratched area in serum-free medium, suggesting that nicotine does not induce cell migration when acting alone. Subsequently, it was examined whether nicotine affects cell migration induced by a pro-migratory stimulus. HGF, which has been demonstrated to be the major pro-migratory stimulus for epithelial cells, including A549 cells, was utilized for this purpose (19-21). As expected, HGF treatment increased cell migration towards the scratched area (Fig. 1). When 0.1 and $1 \mu \mathrm{M}$ nicotine was added to the HGF-treated cells, the cell migratory activity significantly increased $(\mathrm{P}<0.01$; Fig. $1 \mathrm{C})$. Such results suggest that nicotine does not induce cell migration working alone, but that it instead promotes the cell migratory response to the pro-migratory stimulus HGF.

Nicotine promotes HGF-induced cell migration through $\alpha 7-n A c h R$. To determine whether the effect of nicotine in promoting HGF-induced cell migration is mediated by activation of the nicotine receptor, the A549 cells were pretreated with the $\alpha 7-n$ AchR antagonist, methyllycaconitine, prior to adding nicotine to the HGF-treated cells. As presented in Fig. 2, pretreatment with methyllycaconitine abrogated the effect of nicotine in promoting the cell migration induced by HGF treatment $(\mathrm{P}<0.05)$. These results suggest that nicotine enhances the pro-migratory effect of HGF on A549 cells through the activation of $\alpha 7-n$ AchR-mediated signaling pathways.

Nicotine promotes HGF-induced cell migration by potentiating PI3K/Akt activation. HGF binds to and activates its only 
A

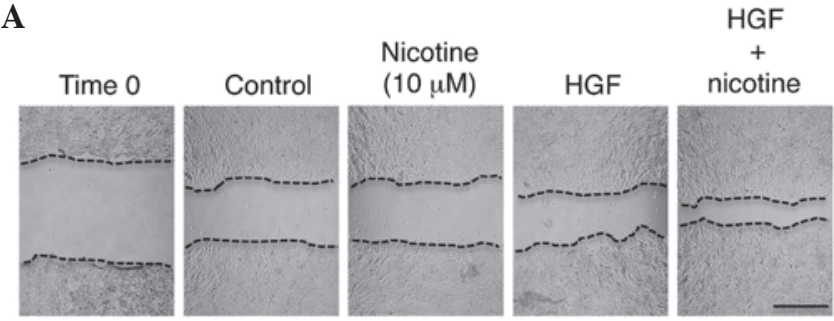

B

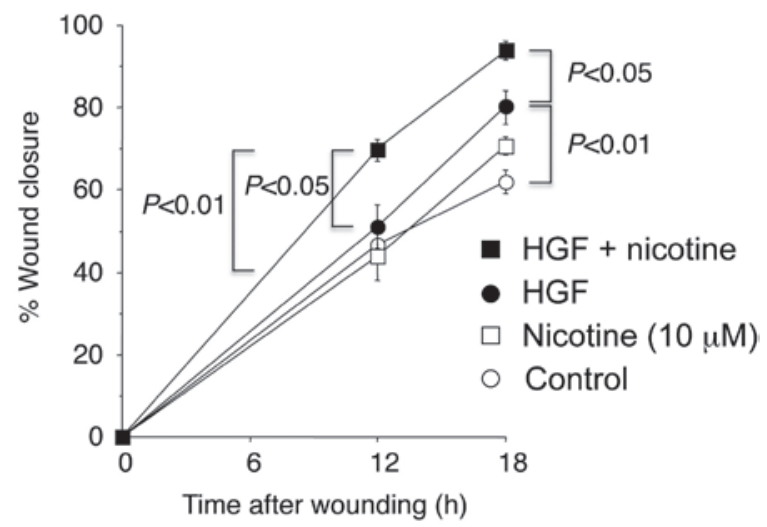

C

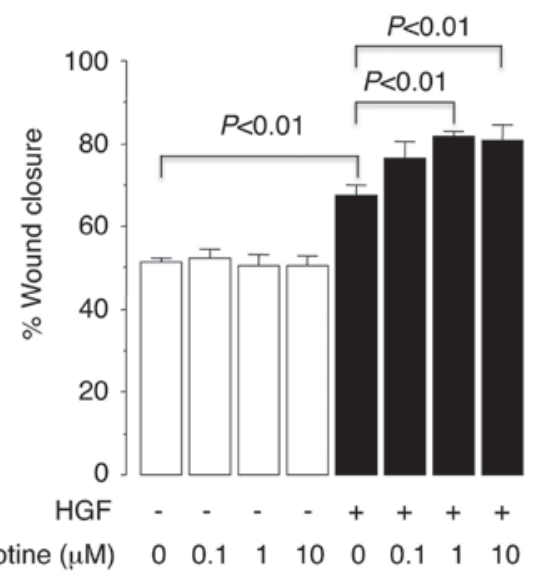

Figure 1. Nicotine promotes migration of HGF-treated A549 cells. A549 cell monolayers were scratched and subsequently incubated in serum-free nicotine-containing medium $(0.1,1$ and $10 \mu \mathrm{M})$, either in the presence or absence of HGF (20 ng/ml). (A) Representative photomicrographs of cell monolayers $18 \mathrm{~h}$ after wounding. Broken lines indicate the leading edge of the wound repopulating cells. Scale bar, $500 \mu \mathrm{m}$. (B) Time-course of the cell migration. The migration of cells towards the wounds was expressed as the percentage of wound closure. The data points represent the mean \pm SEM of 6 wells. (C) Dose-dependent effect of nicotine on cell migration $18 \mathrm{~h}$ post-wounding. The data points represent the mean \pm SEM of 6 wells. HGF, hepatocyte growth factor.

known receptor, c-Met, a receptor tyrosine kinase that mediates downstream signaling cascades (20-23). Results obtained thus

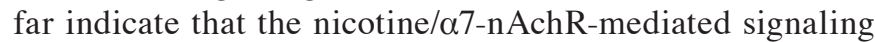
pathway interacts with the $\mathrm{HGF} / \mathrm{c}-$ Met-mediated signaling pathway. The downstream signals of $\mathrm{HGF} / \mathrm{c}-\mathrm{Met}$ include ERK1/2 and PI3K/Akt $(20,21)$. The current study therefore investigated the potential involvement of these downstream signals in the mediation of HGF-induced cell migration. It was demonstrated that pretreatment of the cells with the

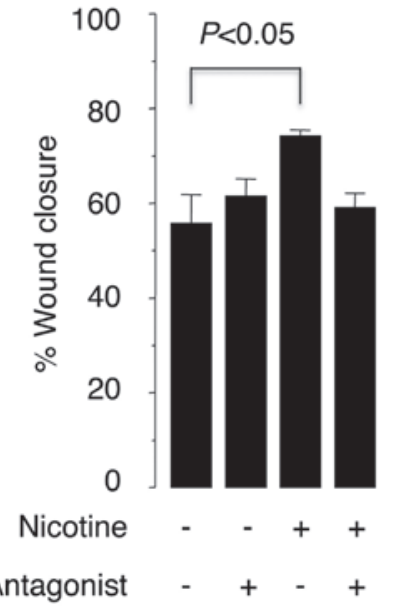

Figure 2. Nicotine promotes HGF-induced cell migration through the activation of $\alpha 7-n A c h R$. A549 cells were pretreated for $1 \mathrm{~h}$ with the $\alpha 7-\mathrm{nAchR}$ antagonist, methyllycaconitine $(10 \mu \mathrm{M})$, prior to inflicting the scratch. The cells were subsequently incubated for $18 \mathrm{~h}$ in serum-free medium, containing or lacking $10 \mu \mathrm{M}$ nicotine in the presence of of $20 \mathrm{ng} / \mathrm{ml} \mathrm{HGF}$. The bars represent the mean \pm standard error of the mean of 6 wells. HGF, hepatocyte growth factor; SEM, standard error of the mean; $\alpha 7-n A c h R, \alpha 7$-nicotine acetylcholine receptor.

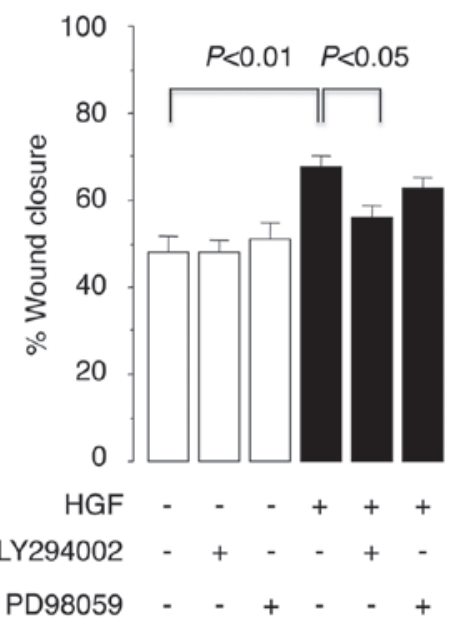

Figure 3. Effects of PI3K and ERK1/2 inhibition on HGF-induced cell migration. A549 cells were pretreated with a pharmacological inhibitor of PI3K, LY294002, or ERK kinase, PD98059, for $1 \mathrm{~h}$. The cells were subsequently scratched and incubated for $18 \mathrm{~h}$ in serum-free medium, either containing or lacking $20 \mathrm{ng} / \mathrm{ml} \mathrm{HGF}$. The bars represent the mean \pm standard error of the mean of 6 wells. PI3K, phosphoinositide kinase-3; ERK, extracellular signal-related kinase; HGF, hepatocyte growth factor.

PI3K inhibitor, LY294002, significantly inhibited the effect of HGF in promoting cell migration $(\mathrm{P}<0.05$; Fig. 3$)$. Similar results were observed following pretreatment of the cells with the MEK inhibitor, PD98059, although the effect was not statistically significant (Fig. 3). These results suggest that the activation of PI3K/Akt is required for $\mathrm{HGF} / \mathrm{c}-$ Met-mediated downstream signaling events that result in A549 cell migration.

Effect of nicotine on the activation of PI3K/Akt. As previously reported (21), HGF treatment induced the phosphorylation of Akt in the A549 cells ( $\mathrm{P}<0.05$; Fig. 4A). Nicotine alone did not induce Akt phosphorylation, but it was noted that it enhanced 
A

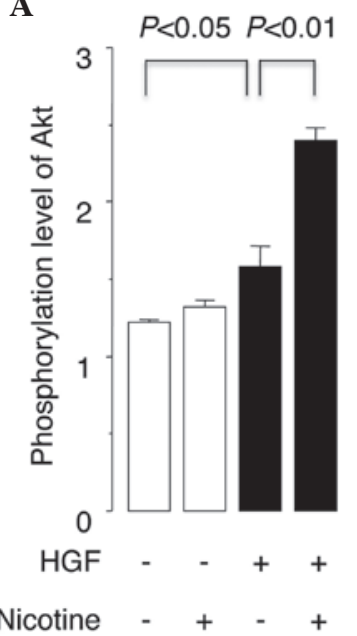

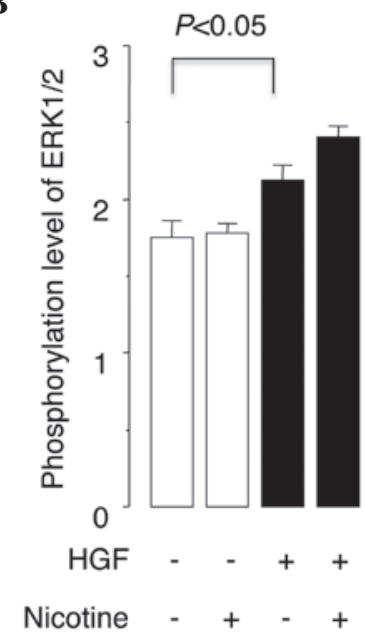

Figure 4. Effects of nicotine on the phosphorylation of Akt and ERK1/2. A549 cells were incubated for $2 \mathrm{~h}$ in a serum-free medium containing $10 \mu \mathrm{M}$ nicotine, either in the presence or absence of $20 \mathrm{ng} / \mathrm{ml}$ HGF. Protein phosphorylation was detected using either (A) the phospho-AKT antibody or (B) the phospho-ERK1/2 antibody, from Fast Activated Cell-based ELISA kits. The protein phosphorylation levels were corrected for the cell numbers. The bars represent the mean \pm standard error of the mean of 6 wells. Akt, protein kinase B; ERK, extracellular signal-related kinase; HGF, hepatocyte growth factor.

the HGF-induced phosphorylation of Akt when compared with HGF alone $(\mathrm{P}<0.01)$. While HGF treatment also significantly induced the phosphorylation of ERK1/2 $(\mathrm{P}<0.05$; Fig. 4B), nicotine had no such effect on the phosphorylation level of ERK1/2 in either the HGF-treated or HGF-untreated cells. When combined, these results indicate that nicotine enhances HGF-induced cell migration through the activation of $\alpha 7-n$ AchRs, whilst also amplifying the HGF-induced activation of PI3K/Akt.

\section{Discussion}

During the present study, it was demonstrated that nicotine does not induce A549 cell migration alone, but that it does promote HGF-induced cell migration. Furthermore, it was also observed that the promotion of HGF-induced cell migration by nicotine mediated its binding to $\alpha 7-\mathrm{nAchR}$, and that as a result, the HGF-mediated PI3K/Akt signaling pathway is amplified. Such results indicate that nicotine is not a pro-migratory factor by itself, but that it instead enhances the cell-migratory responsiveness to the pro-migratory stimulus of HGF.

The findings that nicotine did not induce migration in quiescent A549 cancer cells in the absence of HGF is inconsistent with the results of previous studies, which observed that nicotine itself exerts a pro-migratory effect in various types of cancer cells $(10,12-14,16,17)$. The reasoning behind the inconsistent results between the present study and previous studies remains uncertain, however, it may be attributed to differences in the experimental protocol. The current study performed a wound healing assay in serum-free medium following $48 \mathrm{~h}$ of serum starvation, while in previous studies, the assay was performed in serum-supplemented medium $(10,12,14,17)$, and/or without serum starvation prior to inflicting the scratch (16). Since serum may contain

pro-migratory factors, including $\mathrm{HGF}$, serum contamination during the wound healing assay may therefore potentiate cell migration following the addition of nicotine.

HGF serves a critical role in cancer progression by upregulating cell proliferation, migration, invasion and angiogenesis (22). Elevated HGF and c-Met expression, as well as numerous c-Met mutations, have been reported in human cancer, including lung cancer, and have been demonstrated to be correlated with a poor prognosis (22). Accordingly, c-Met has been proposed as a possible therapeutic target for the treatment of lung cancer (23). However, the $\mathrm{HGF} / \mathrm{c}-$ Met-mediated signaling pathway is complex. Recent evidence suggests that c-Met cross-talks with other receptors, including the epidermal growth factor receptor and the recepteur d'origine nantais (23). To the best of our knowledge, the present study provides evidence of crosstalk between $\mathrm{HGF} / \mathrm{c}-\mathrm{Met}$ and nicotine/n AchR for the first time.

The current study demonstrated that the PI3K inhibitor, LY294002, inhibited cell migration that had been induced by HGF treatment, corroborating the results of previous studies and establishing that PI3K/Akt is an essential mediator downstream of HGF/c-Met $(20,22,23)$. It was also demonstrated that Akt was activated by HGF, but not by nicotine. However, Akt activation by HGF treatment was enhanced when the cells were co-treated with nicotine. These findings suggest that nicotine upregulates the HGF-induced activation of the PI3K/Akt pathway, leading to the enhancement of the cell migratory response to HGF.

nACHRs mediate a number of the effects exerted by nicotine (24). Among them, $\alpha 7-n$ AchR has been demonstrated to be expressed in cancer cells, including lung cancer A549 cells $(24,25)$. Lending support to the demonstration of cross-talk between nicotine/ $\alpha 7-n$ AchR and HGF/c-Met in the present study, previous studies have established that nicotine/ $\alpha 7-n A c h R$ cross-talks with the downstream signaling of various growth factors, including epithelial cell growth factor, basic fibroblast growth factor and vascular endothelial cell growth factor, promoting cancer cell proliferation $(4,17,24)$. However, the mechanisms underlying signal crosstalk are complex. The binding of nicotine to $\mathrm{nAchR}$ increases $\mathrm{Ca}^{2+}$ influx, thereby potentially modulating $\mathrm{Ca}^{2+}$-dependent signal transduction $(11,24)$. Nicotine may also increase the expression of receptor tyrosine kinases and the recruitment of growth/migration-associated factors to the surface of cells $(17,24)$. The effects of nicotine demonstrated in the present study are unlikely to be due to direct activation of PI3K/Akt by nicotine, as nicotine alone did not induce phosphorylation of Akt in the serum-free condition in the absence of HGF. It is conceivable that nicotine/ $\alpha 7-n$ AchR may act in synergy with the HGF/c-Met-induced pathway, elevating the sensitivity of cancer cells to HGF, and thereby promoting cell migration.

In the present study, nicotine enhanced HGF-induced cell migration at concentrations of 1 and $10 \mu \mathrm{M}$. This range of nicotine concentrations reasonably mimics the nicotine concentrations observed in vivo in humans. While plasma concentrations of nicotine in chronic smokers are reported to range between 0.01 and $1 \mu \mathrm{M}(26)$, lung tissue concentrations of nicotine may be much higher, due to the direct contact of the lung tissue with nicotine in cigarette smoke. 
In conclusion, the current study demonstrated that nicotine treatment potentiates the migratory responsiveness of lung cancer cells to HGF. Cumulative evidence suggests that nicotine may have a broad spectrum of tumor-promoting activities, which include increasing cell proliferation, invasion, EMT and angiogenesis (4). The present study provides an insight into the mechanism of the promotion of tumor cell activities initiated by nicotine, by demonstrating how nicotine-modulated growth factor-mediated signal transduction leads to increased cell motility, a vital step in cancer invasion and metastasis. Furthermore, the results also provide evidence of the inhibition of $\alpha 7-n A c h R$ as a potential therapeutic target.

\section{References}

1. World Health Organization: World Cancer Report 2014 www.iarc.fr/en/publications/books/wcr/wcr-order.php. Accessed October 30, 2015.

2. American Cancer Society. Cancer facts and figures. 2014. http:// www.cancer.org/acs/groups/content/@research/documents/ webcontent/acspc-042151.pdf. Accessed October 30, 2015.

3. Hecht SS: Lung carcinogenesis by tobacco smoke. Int J Cancer 131: 2724-2732, 2012.

4. Warren GW and Singh AK: Nicotine and lung cancer J Carcinog 12: 1, 2013.

5. Videtic GM, Stitt LW, Dar AR, Kocha WI, Tomiak AT, Truong PT, Vincent MD and Yu EW: Continued cigarette smoking by patients receiving concurrent chemoradiotherapy for limited-stage small-cell lung cancer is associated with decreased survival. J Clin Oncol 21: 1544-1549, 2003.

6. Andreas S, Rittmeyer A, Hinterthaner M and Huber RM: Smoking cessation in lung cancer-achievable and effective. Dtsch Arztebl Int 110: 719-724, 2013.

7. Joshu CE, Mondul AM, Meinhold CL, Humphreys EB, Han M, Walsh PC and Platz EA: Cigarette smoking and prostate cancer recurrence after prostatectomy. J Natl Cancer Inst 103: 835-838, 2011.

8. Kenfield SA, Stampfer MJ, Chan JM and Giovannucci E: Smoking and prostate cancer survival and recurrence. JAMA 305: 2548-2555, 2011.

9. Warren GW, Romano MA, Kudrimoti MR, Randall ME, McGarry RC, Singh AK and Rangnekar VM: Nicotinic modulation of therapeutic response in vitro and in vivo. Int J Cancer 131: 2519-2527, 2012.

10. Xu L and Deng X: Protein kinase Ciota promotes nicotine-induced migration and invasion of cancer cells via phosphorylation of micro- and m-calpains. J Biol Chem 281: 4457-4466, 2006.

11. Zhang Q, Tang X,Zhang ZF, Velikina R, Shi S and Le AD: Nicotine induces hypoxia-inducible factor-1alpha expression in human lung cancer cells via nicotinic acetylcholine receptor-mediated signaling pathways. Clin Cancer Res 13: 4686-4694, 2007.

12. Guo J, Ibaragi S, Zhu T, Luo LY, Hu GF, Huppi PS and Chen CY: Nicotine promotes mammary tumor migration via a signaling cascade involving protein kinase $\mathrm{C}$ and CDC42. Cancer Res 68 . 8473-8481, 2008
13. Dasgupta P, Rizwani W, Pillai S, Kinkade R, Kovacs M, Rastogi S, Banerjee S, Carless M, Kim E, Coppola $\mathrm{D}$, et al: Nicotine induces cell proliferation, invasion and epithelial-mesenchymal transition in a variety of human cancer cell lines. Int J Cancer 124: 36-45, 2009.

14. Lien YC, Wang W, Kuo LJ, Liu JJ, Wei PL, Ho YS, Ting WC, $\mathrm{Wu} \mathrm{CH}$ and Chang YJ: Nicotine promotes cell migration through alpha7 nicotinic acetylcholine receptor in gastric cancer cells. Ann Surg Oncol 18: 2671-2679, 2011

15. Shi D, Guo W, Chen W, Fu L, Wang J, Tian Y, Xiao X, Kang T, Huang W and Deng W: Nicotine promotes proliferation of human nasopharyngeal carcinoma cells by regulating $\alpha 7 A C h R$, ERK, HIF-1 $\alpha$ and VEGF/PEDF signaling. PLoS One 7: e43898, 2012.

16. Momi N, Ponnusamy MP, Kaur S, Rachagani S, Kunigal SS Chellappan S, Ouellette MM and Batra SK: Nicotine/cigarette smoke promotes metastasis of pancreatic cancer through $\alpha 7$ nAChR-mediated MUC4 upregulation. Oncogene 32: 1384-1395, 2013

17. Khalil AA, Jameson MJ, Broaddus WC, Lin PS and Chung TD: Nicotine enhances proliferation, migration, and radioresistance of human malignant glioma cells through EGFR activation. Brain Tumor Pathol 30: 73-83, 2013.

18. Tsuji T, Aoshiba K, Itoh M, Nakamura H and Yamaguchi K: Hypercapnia accelerates wound healing in endothelial cell monolayers exposed to hypoxia. Open Respir Med J 7: 6-12, 2013.

19. Stoker M, Gherardi E, Perryman M and Gray J: Scatter factor is a fibroblast-derived modulator of epithelial cell mobility. Nature 327: 239-242, 1987.

20. Ponzetto C, Bardelli A, Zhen Z, Maina F, dalla Zonca P, Giordano S, Graziani A, Panayotou G and Comoglio PM: A multifunctional docking site mediates signaling and transformation by the hepatocyte growth factor/scatter factor receptor family. Cell 77: 261-271, 1994

21. Forte G, Minieri M, Cossa P, Antenucci D, Sala M, Gnocchi V, Fiaccavento R, Carotenuto F, De Vito P, Baldini PM, et al: Hepatocyte growth factor effects on mesenchymal stem cells: Proliferation, migration, and differentiation. Stem Cells 24: 23-33, 2006.

22. Maroun CR and Rowlands T: The Met receptor tyrosine kinase: A key player in oncogenesis and drug resistance. Pharmacol Ther 142: 316-338, 2014.

23. Sadiq AA and Salgia R: MET as a possible target for non-small-cell lung cancer. J Clin Oncol 31: 1089-1096, 2013.

24. Niu XM and Lu S: Acetylcholine receptor pathway in lung cancer: New twists to an old story. World J Clin Oncol 5: 667-676, 2014

25. Dasgupta P, Kinkade R, Joshi B, Decook C, Haura E and Chellappan S: Nicotine inhibits apoptosis induced by chemotherapeutic drugs by up-regulating XIAP and survivin. Proc Natl Acad Sci USA 103: 6332-6337, 2006.

26. Benowitz NL: Drug therapy. Pharmacologic aspects of cigarette smoking and nicotine addiction. N Engl J Med 319: 1318-1330, 1988 